\title{
ROLLOVER WARNING FOR ARTICULATED HEAVY VEHICLES BASED ON A TIME-TO-ROLLOVER METRIC
}

\author{
Bo-Chiuan Chen ${ }^{1}$ \\ Department of Vehicle Engineering \\ National Taipei University of Technology
}

\author{
Huei Peng ${ }^{2}$ \\ Department of Mechanical Engineering \\ University of Michigan
}

\begin{abstract}
A Time-To-Rollover (TTR) metric is proposed as the basis to assess rollover threat for an articulated heavy vehicle. The TTR metric accurately "counts-down” toward rollover regardless of vehicle speed and steering patterns, so that the level of rollover threat is accurately presented. There are two conflicting requirements in the implementation of TTR. On the one hand, a super-real-time model is needed. On the other hand, the TTR predicted by this model needs to be accurate enough under all driving scenarios. An innovative approach is proposed in this paper to solve this dilemma and the design process is illustrated in an example. First, a simple yet reasonably accurate yaw/roll model is identified. A Neural Network (NN) is then developed to mitigate the accuracy problem of this simple model. The NN takes the TTR generated by the simple model, vehicle roll angle, and change of roll angle to generate an enhanced NN-TTR index. The NN was trained and verified under a variety of driving patterns. It was found that an accurate TTR is achieved across all the driving scenarios we tested.
\end{abstract}

\section{INTRODUCTION}

The safe operation of articulated heavy vehicles has been a major concern due to their large size and weight. Recent statistics on truck and bus crashes [1] show that rollover occurred in 53.8\% of the first harmful event of noncollision type crashes. Furthermore, the fatality rate of rollover accidents was found to be significantly higher than non-rollover cases. Therefore, active counter-measures for rollover crashes are crucial for safe highway operations. The research results to be reported in this paper focus on the development of a rollover threat metric utilizing advanced techniques to improve the vehicle status assessment. The developed metric can be used as the basis for rollover warning or rollover prevention systems.

\footnotetext{
${ }^{1}$ Assistant Professor, Department of Vehicle Engineering, National Taipei University of Technology, Taipei 106, TAIWAN, 886-2-27712171 Ext. 3622, bochen@ntut.edu.tw.

${ }^{2}$ Corresponding author. Associate Professor, Department of Mechanical Engineering, University of Michigan, Ann Arbor, MI 48109-2133, 734936-0352, hpeng@umich.edu.
} 
Driver's awareness of rollover on existing (non-instrumented) vehicles depends on vehicle type, load position, and torsional stiffness of the trailer, and many other vehicle parameters [2]. A torsionally flexible trailer results in poor roll feedback to the driver, and is found to be a major issue for flatbed trucks. The fact that the tractor has a relatively low C.G. height almost separates the driver from the trailer due to the small tractor roll motion. The driver often remains unaware of the rollover instability at the trailer and fails to take corrective actions in a timely fashion until it is too late. By monitoring the dynamic states of the articulated heavy vehicle, an accurate and timely warning could be issued for impending rollover threat.

Most of the existing rollover warning algorithms are based on lateral acceleration or roll angle threshold values. Rakheja [3] proposed an early warning safety monitor in 1990. First, under static cornering maneuvers, the magnitude of lateral acceleration at inner-tire lift-off is defined as the threshold value. Under driving conditions, a warning is then issued whenever the measured lateral acceleration exceeds this threshold. The same idea was also used to define a threshold for the semi-trailer roll angle. Preston-Thomas [4] used the lateral load transfer ratio (LTR) to indicate rollover threat. LTR can vary from zero when the loads carried by the left tires and right are identical, to a maximum value of one when the tires lift off and the lateral acceleration reaches the threshold. Freedman et al. [5] proposed a rollover advisory sign on highway exit ramps. When the speed of the truck is higher than the advised speed limit, which is determined based on the curvature of the exit ramp, a warning signal is triggered. In their research, the warning signal did not use additional information to identify each truck's threshold. McGee et al. [6,7] proposed a warning system, which is similar to the one of [5]. However, their system detects the type, speed, weight, and height of the truck and identifies the acceleration threshold of each vehicle from a look-up table. Ervin et al. [8] proposed a rollover stability advisor (RSA) system. RSA determines the rollover acceleration threshold based on real-time measurements of the status of the vehicle. The required measurements include forces and moments at the fifth wheel and a set of roll motion variables. Three acceleration threshold values are calculated dynamically to determine the rollover threshold level.

Nalecz et al. proposed an energy based function named Rollover Prevention Energy Reserve (RPER) in 1987 [9-11]. RPER is defined as the difference between the energy needed to bring the vehicle to its tip-over position and the rotational kinetic energy, which can be transferred into the gravitational potential energy to lift the vehicle. RPER remains positive for non-rollover cases. When it becomes negative, a rollover will occur if nothing is done to 
take energy out of the roll mode. A special advantage of RPER is that the same concept can be applied to both maneuver induced and tripped rollover incidents.

The concepts mentioned above were based on acceleration, roll angle or energy threshold values which are estimated from the information at a fixed time. In analogy, it is like taking a still picture of a dynamic system and uses the information (frozen in time) to determine the rollover threat. Apparently, a method which covers factors over a longer time horizon, particularly into the future, could give us a better perspective. Furthermore, the distance away from these threshold levels is not an intuitive measure. Therefore, we propose a Time-To-Rollover (TTR) metric as the basis to assess rollover threat. Ideally, a TTR metric will accurately "count down" toward rollover under a wide range of vehicle speeds and steering patterns, so that the level of rollover threat is accurately indicated. There are two conflicting requirements to implement TTR in real-time. On one hand, a super-real-time (much faster-than-real-time) vehicle model is needed. For example, in order to predict a TTR of (up to) 3 sec, one needs to predict vehicle response in the next $3 \mathrm{sec}$ repeatedly. If TTR is updated every 50ms, the vehicle model needs to be 60 times faster than real-time. On the other hand, the TTR predicted by this model needs to be accurate enough under all driving scenarios, so that the rollover warning system can make good warning decisions. An innovative approach is proposed in this paper to solve this dilemma.

The remainder of this paper is organized as follows: the TTR metric is defined and presented in Section 2. The Neural Network (NN) structure used to produce "desired" TTR response is also discussed. The complex and simple models used in this research are presented in Section 3. In Section 4, a set of rollover and non-rollover maneuvers are designed to cover a wide variety of operation conditions. Simulation results of the overall TTR computations are presented in Section 5. Finally, conclusions are made in Section 6.

\section{TIME-TO-ROLLOVER (TTR) METRIC}

Many existing rollover warning algorithms do not have the capability to predict the impending rollover danger in the future. The predictive capability is necessary in order to give drivers more time to react to the rollover threat and avoid the rollover accident. Motion prediction techniques have been used for vehicle active safety systems because they can predict vehicle motions under all driving scenarios. Time-To-Collision (TTC) and Time To LaneCrossing (TLC) are two major applications of motion prediction techniques. TTC utilizes vehicle longitudinal dynamics for motion prediction. Hayward [12] defined TTC as the time required for two vehicles to collide if they 
continue at their present speed and stay on the same path. TLC utilizes vehicle yaw dynamics for motion prediction. Lin [13-16] defined TLC as the time remaining until the vehicle CG crosses the lane edge, assuming the steering wheel angle remains constant.

These techniques are used to help develop the TTR metric for assessing the rollover threat. Presumably, the TTR metric should be accurate across a wide array of rollover conditions. If TTR does not perform well, an artificial NN will be added to process the model-based TTR.

\subsection{Definition of Time-To-Rollover}

Tire lift-off is defined as the unacceptable rollover event in this paper. To be more precise, the term "Time-ToRollover” actually means "Time-To-Wheel-lift-off.” This definition will not result in any major change in the overall algorithm development. A more aggressive or more conservative roll event definition can be used and the design process to be described below will remain the same.

After a "rollover" (or, wheel-lift-off) has occurred, a true-TTR can be computed in an after-the-fact manner. In other words, whenever the vehicle roll angle exceeds the defined threshold value, we can roll back the clock and define a point $0.5 \mathrm{sec}$ before this tire-lift-off incident to have a "true-TTR" of $0.5 \mathrm{sec}$, i.e. the vehicle rolls over 0.5 sec later. This is like drawing a straight line with slope -1 on the time-TTR plot from the rollover instant (see Fig. 1). Ideally, if we can re-construct the TTR in real-time and in a predictive manner, the severity of the rollover threat can be accurately represented and reported.

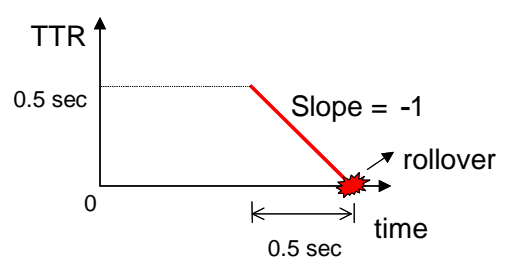

Fig. 1 Definition of TTR

The TTR metric is defined as following: assuming the input steering angle stays fixed at its current level in the foreseeable future, the time which takes for the vehicle sprung mass to reach its critical roll angle is defined as TTR. The flow chart of the TTR calculation algorithm is shown in Fig. 2. The vehicle model takes the necessary initial conditions and driver's steering input at each sampling time. Then the algorithm integrates the model for up to X sec into the future, by assuming constant steering during the integration time horizon. Under normal driving conditions, the TTR is usually very large and may even approach infinity. For example, if the driver is driving 
straight, there is no roll motion at all and the TTR is approaching infinity. For implementation considerations, we saturate the TTR at X sec. In other words, we will only integrate the vehicle model for up to X sec. If the vehicle does not roll over in the foreseeable future, the TTR is determined to be X sec. The roll angle threshold can be specified differently for different vehicles, or for different aggressiveness of roll motion predictions.

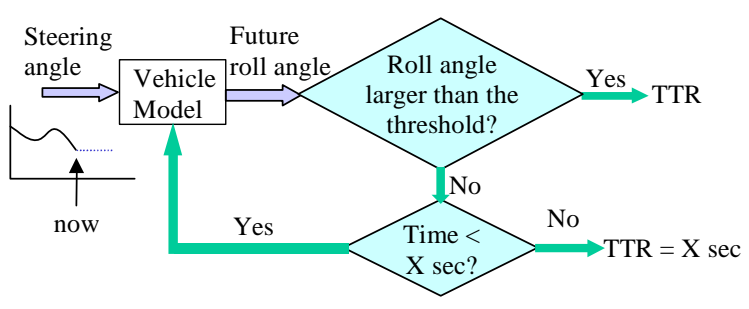

Fig. 2 Flow chart for the TTR calculation

The vehicle model can be either a nonlinear complex or a linear simple model. In order to predict a TTR of (up to) 3 seconds, one needs to predict vehicle response quickly. As mentioned in Section 1, if TTR is updated every 50 ms, the vehicle model needs to be 60 times faster than real-time. Therefore, we can only afford to use the simple model to calculate the TTR metric. The simple model can be obtained by system identification techniques or Newtonian/Lagrangian approaches (deriving the equations of motion).

\section{$\underline{2.2 \text { Neural Network TTR }}$}

If the simple model does not capture enough of the dynamics of the full nonlinear complex model, the TTR from the simple model (simple TTR) might not be accurate enough. When the simple TTR fails to satisfy the desired TTR characteristic, which is a straight line of slope $=-1$ for rollover cases and a straight line of $\mathrm{X}$ sec for non-rollover cases (on a time-TTR plot), a NN is introduced to correct the error between the simple TTR and the desired (true) TTR. The structure of the NN is shown in Fig. 3. In additional to the simple TTR, the NN also uses the vehicle roll angle and the change of roll angle to produce the NN-TTR.

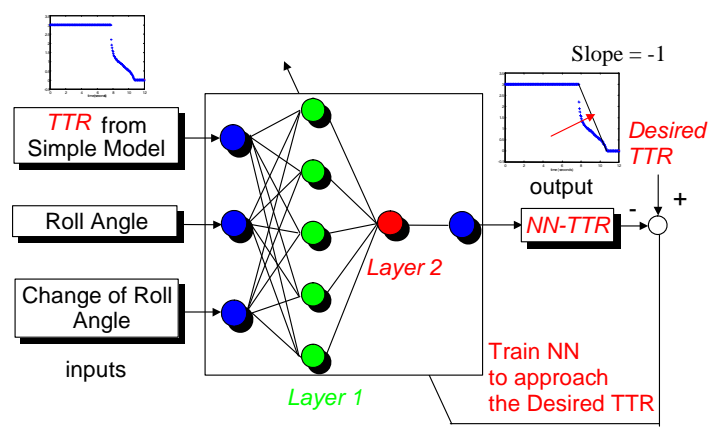

Fig. 3 Structure of the Neural Network 
The NN is trained by using training data files with and without rollover incidents, and then verified by using evaluation data files with and without rollover incidents. If the NN is not trained with all maneuvers, it might not perform as expected for the unseen maneuvers. However, it is impossible to train the NN for all the maneuvers. The training set should include three types of maneuvers: mild, bad, and worst-case (see Fig. 4). The motivation for using the worst-case $[17,18]$ maneuver is to help train the $\mathrm{NN}$ to be as complete as possible so that the $\mathrm{NN}$ can handle the unseen maneuvers as well. After training, the NN will be used to generate a corrected TTR (NN-TTR) across all vehicle speeds and steering patterns.

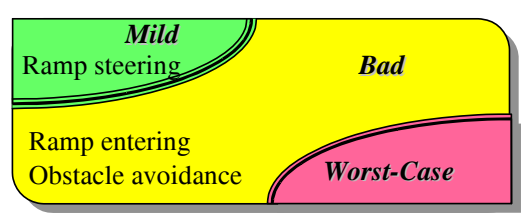

Fig. 4 Severity of the NN training maneuvers

\section{MODELING}

\section{$\underline{3.1 \text { Complex model }}$}

In this paper, the simulation response of the ArcSim software will be assumed to be the true vehicle output. ArcSim is a simulation program developed at the Automotive Research Center (ARC) of the University of Michigan for studying the braking and handling response of the M916A1/M870A2 Army Truck. This truck has a three-axle tractor and a three-axle semi-trailer. In addition to the vehicle dynamics Simulation and Graphic User Interface (SGUI, shown in Fig. 5), ArcSim also provides a plotter and an animator to visualize simulation results. The ArcSim software runs under Windows 95/98/2000/NT and is a freeware available at the following link: http://arc.engin.umich.edu/sw_distri/ARCSIM/arcsim.html.

The ArcSim model has 21 degrees of freedom (DOF). Three translational (longitudinal, lateral, and vertical) and three rotational (roll, pitch, and yaw) motions of the tractor sprung mass are considered. The semi-trailer rotates freely with respect to the tractor in the yaw and pitch direction, but otherwise its motion is constrained by a fifth wheel, which is a hitch connecting the tractor and the semi-trailer. The roll and jounce (vertical) motions of six wheel axles are also modeled and each of the twelve wheels spins independently. This model has a computation efficiency of about 1.0 (i.e. 1 sec computational time for 1 sec simulation) on a Pentium 600MHz PC and can be 
simulated in real-time, but not super-real-time as mentioned in previous sections. Since a super-real-time vehicle model is crucial for TTR calculation, a simple model needs to be developed.

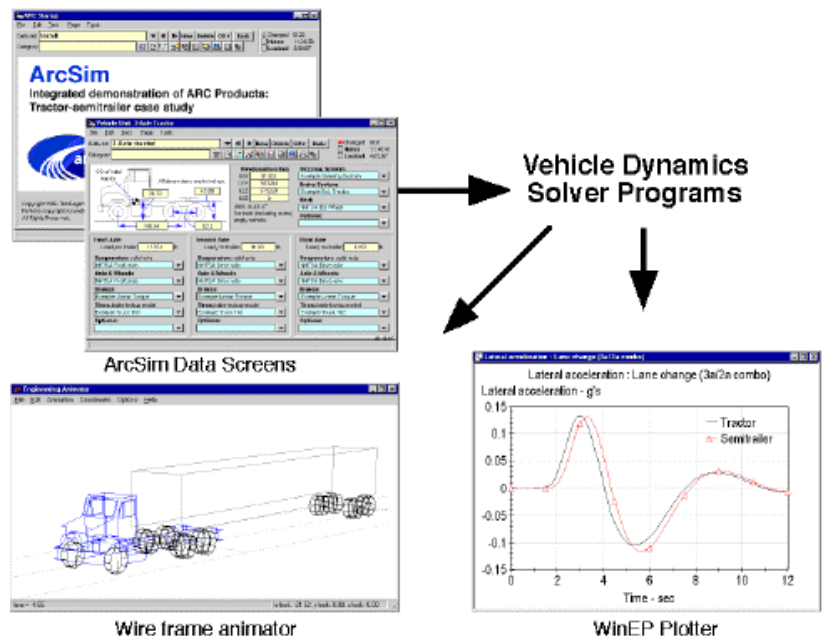

Fig. 5 SGUI of ArcSim

\section{$\underline{3.2}$ Simple model}

We chose to develop a model that has decoupled yaw and roll dynamics (see Fig. 6). The only input to the model is the steering angle. Other inputs, such as the driver's braking and road super-elevation are neglected. The output of the yaw model is the lateral acceleration, and outputs of the roll model include the roll angle(s) and roll rate(s) of the vehicle. Since the yaw and roll models are actually coupled, the proposed decoupled yaw-roll model may produce less accurate results than an integrated yaw-roll model. However, this simple structure is superior in two aspects: easy model construction and fast computation.

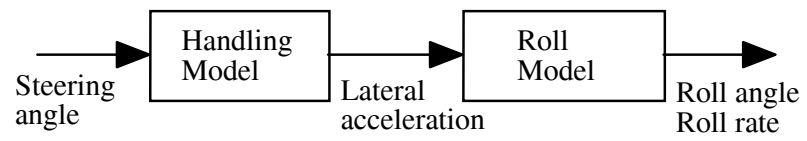

Fig. 6 Structure of the simple model

The structure of the yaw model depends on the selection of the roll model. Therefore, the roll model needs to be determined first. We constructed four simple roll models (see Fig. 7), which include 0, 2, 4, and 8 state variables, respectively. This set of models represents a progressively closer approximation of the ArcSim model. Model 'A' (zero DOF) is a single-unit rigid vehicle. In other words, neither suspension/tire compliance nor articulation is included in the model. In Model 'B' (1 DOF), tire compliance is included. Suspension compliance is added to Model 'B' to obtain Model 'C’ (2 DOF). Finally, the vehicle articulation is recognized in Model 'D' (4 DOF). 

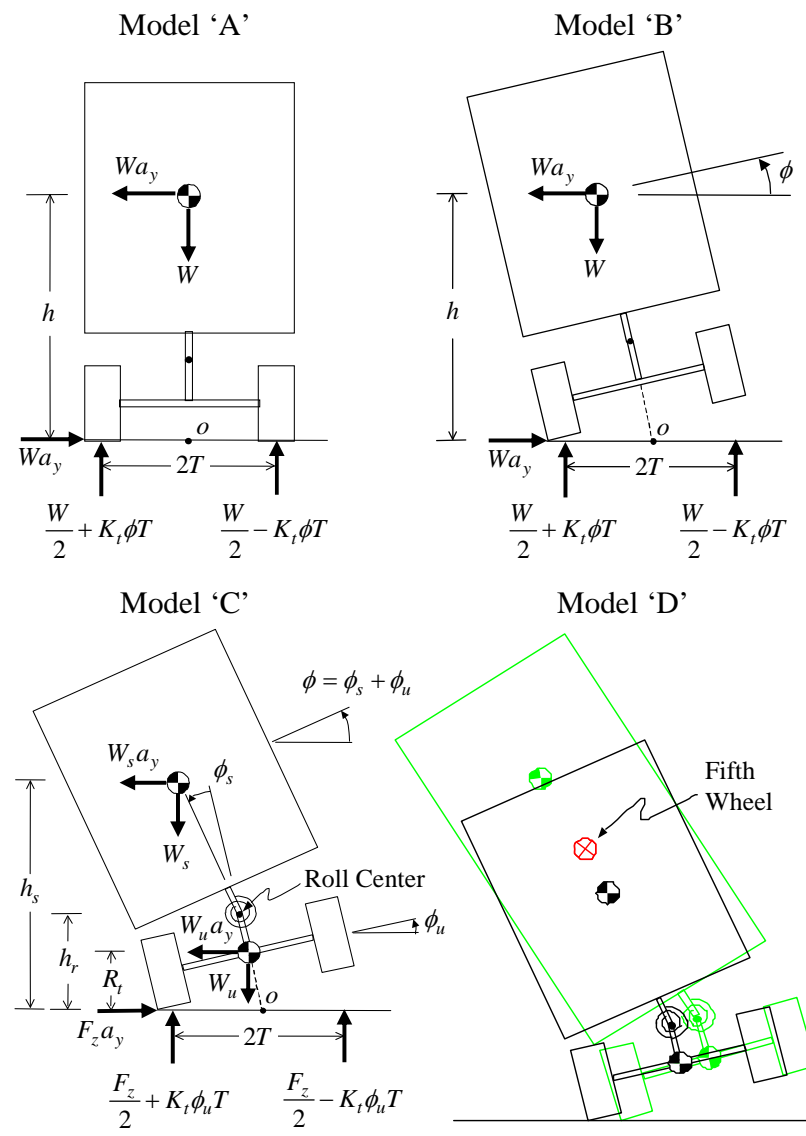

Model 'D'

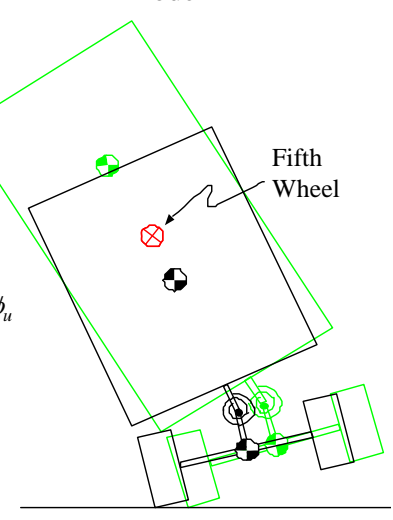

Fig. 7 Configuration of the four simple roll models

Detail equations of these four roll models are presented in the Appendix A. Here we will just summarize the steady-state roll characteristics of these models. When the steering input is slowly increased, each of these four models will predict lateral acceleration and roll angle thresholds of the semi-trailer for the tire lift-off condition. These predicted threshold values are listed in Table 1 together with the ArcSim simulation results.

Table 1 Summary of axle lift-off threshold values of the semi-trailer

\begin{tabular}{|c|c|c|c|}
\hline & $\begin{array}{c}\text { Lateral } \\
\text { acceleration (g) }\end{array}$ & $\begin{array}{c}\text { Sprung mass } \\
\text { roll angle (deg) }\end{array}$ & $\begin{array}{c}\text { Unsprung mass } \\
\text { roll angle (deg) }\end{array}$ \\
\hline Model A & 0.4458 & 0.0000 & 0.0000 \\
\hline Model B & 0.4189 & 1.5448 & 1.5448 \\
\hline Model C & 0.3992 & 3.9834 & 3.2811 \\
\hline Model D & 0.2899 & 3.1442 & 3.2460 \\
\hline ArcSim & 0.2763 & 3.2869 & 3.4051 \\
\hline
\end{tabular}

It can be seen that at just $4 \mathrm{DOF}$, Model $\mathrm{D}$ is able to predict the steady-state acceleration and roll angle threshold values of the semi-trailer very accurately. Therefore, the roll model shown in Fig. 7 will be a dynamic version of Model D, Dynamic Model D (its detailed derivation is also shown in Appendix A). In deriving the 
dynamic model, a semi-static approach is used, i.e. static in the yaw plane, and dynamic in the roll plane. The time derivatives of the roll angles are no longer assumed to be zero. Thus the damping and inertia terms need to be defined and included in the dynamic equations. Lateral accelerations are assumed the same for the tractor and the semi-trailer. Because the roll model includes two vehicle units (tractor and semi-trailer), the yaw model will be described by the "bicycle-towing-unicycle" configuration shown in Fig. 8.

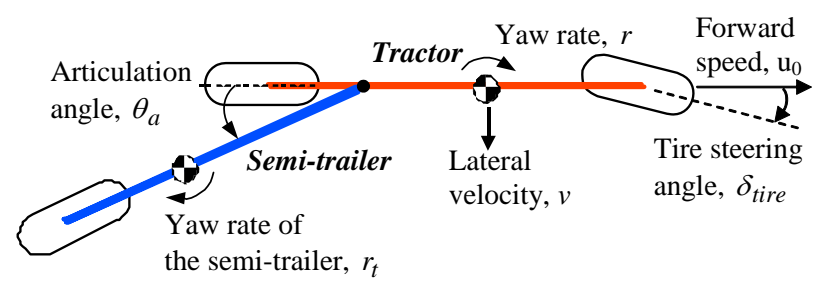

\section{Fig. 8 Bicycle-towing-unicycle yaw model of an articulated heavy truck}

The yaw model requires 4 state variables. The structure of the speed-dependent $4^{\text {th }}$ order transfer function from the steering input to the lateral acceleration of the semi-trailer is shown in Eq. (1).

$$
T_{\text {yaw }}(z)=\frac{b_{0} z^{4}+b_{1} z^{3}+b_{2} z^{2}+b_{3} z^{1}+b_{4}}{z^{4}+a_{1} z^{3}+a_{2} z^{2}+a_{3} z^{1}+a_{4}}=\frac{a_{y}}{\delta}
$$

where $a_{y}$ is the lateral acceleration of the semi-trailer and $\delta$ is the hand wheel steering angle. $\delta_{\text {tire }}$ shown in Fig. 8 is equal to $\delta$ divided by the gear ratio of the steering system. $a_{i}$ 's and $b_{i}$ 's are coefficients of the transfer function which are gain scheduled with respect to different vehicle speeds. The lateral acceleration of the semitrailer is used as the input to the roll model (Dynamic Model D). Pseudo-random steering was fed to ArcSim to generate a sequence of input/output response. Then system identification techniques were applied to obtain coefficients of the transfer function.

Example response from the simple decoupled yaw-roll model under an obstacle avoidance maneuver (vehicle speed at $60 \mathrm{mph}$ with obstacle $1 \mathrm{sec}$ ahead) is shown in Fig. 9. It can be seen that the response of the simple model is similar to that of the ArcSim model, but the difference is noticeable. As can be seen from Fig. 9, the difference might arise mainly from the fact that the lateral acceleration response was not predicted accurately. This difference is acceptable because (1) the simple model is computationally about 200 times faster than the ArcSim model and thus can be used for TTR calculation in real-time; and (2) this minor difference in response can be compensated later by a NN and therefore will not cause any performance concern. 

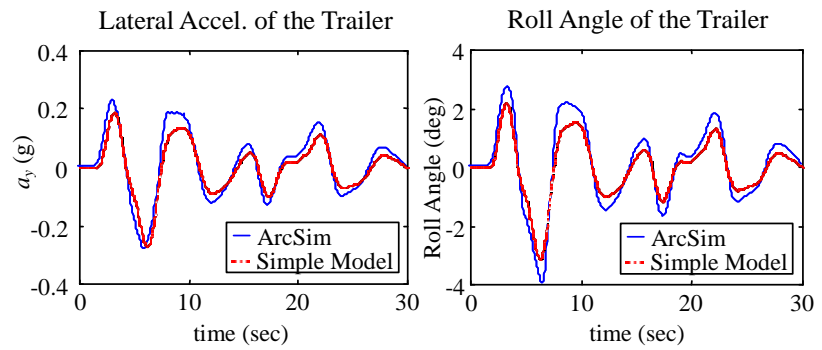

Fig. 9 ArcSim and simple model response under an obstacle avoidance maneuver

\section{MANEUVER SELECTION}

A set of maneuvers was designed to cover a wide array of rollover or near-rollover incidents. These maneuvers can be classified into the following types: ramp steering (i.e., slowly increased steering), ramp entering, obstacle avoidance, and “worst-case” maneuvers (see Fig. 10).
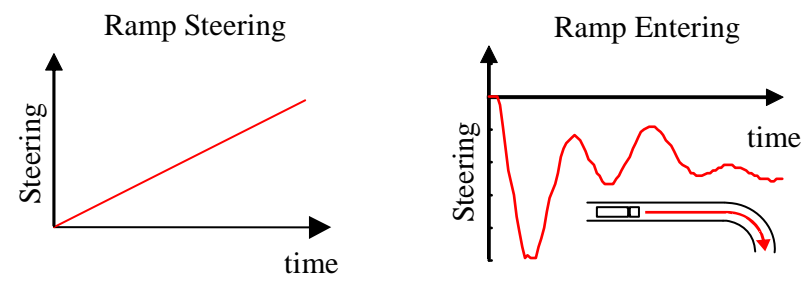

Obstacle Avoidance

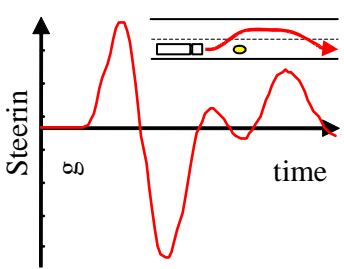

Worst-case Steering

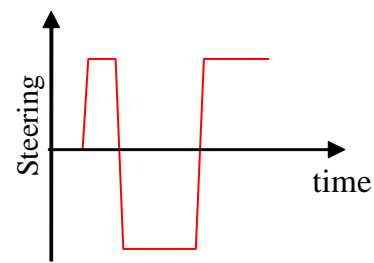

Fig. 10 Four types of maneuvers

Each driving pattern consists of multiple cases such that both rollover and non-rollover maneuvers are included.

The ramp entering and obstacle avoidance maneuvers represent driving patterns responsible for common highway rollover incidents. Ramp steering is a slow but sure way to create rollover under normal driving speed, and represents smoothest possible rollover excitations. The "worst-case" maneuvers were generated from the "worstcase evaluation method” proposed in $[17,18]$, which gave the worst possible input within a given steering bound. This steering maneuver represents the most violent rollover excitations, which would be generated in the event of 
the most unexpected driving situations. By covering this wide array of smooth/bad/worst-case spectrum of rollover incidents in the maneuver design, the performance of the rollover warning algorithm can be better assessed.

Another important fact to notice is that the steering inputs for the ramp entering and obstacle avoidance maneuvers were obtained indirectly by using a STI driver model [19] to which the desired vehicle trajectory was specified. The driver model closed the loop between the environment and the vehicle dynamics. Due to the incompatible simulation environment of the ArcSim and the STI driver model in Matlab/Simulink, the vehicle model were represented by the identified bicycle-towing-unicycle yaw model instead of ArcSim. The generated steering angle was then fed to ArcSim for simulation. Therefore, it is an indirect approach to obtain the steering from the driver model. The driver parameters were tuned with respect to the identified yaw model so that the crossover model principle [20] was satisfied. The block diagram of the driver model is shown in Fig. 11, and the values of the driver parameters are listed in Appendix B.

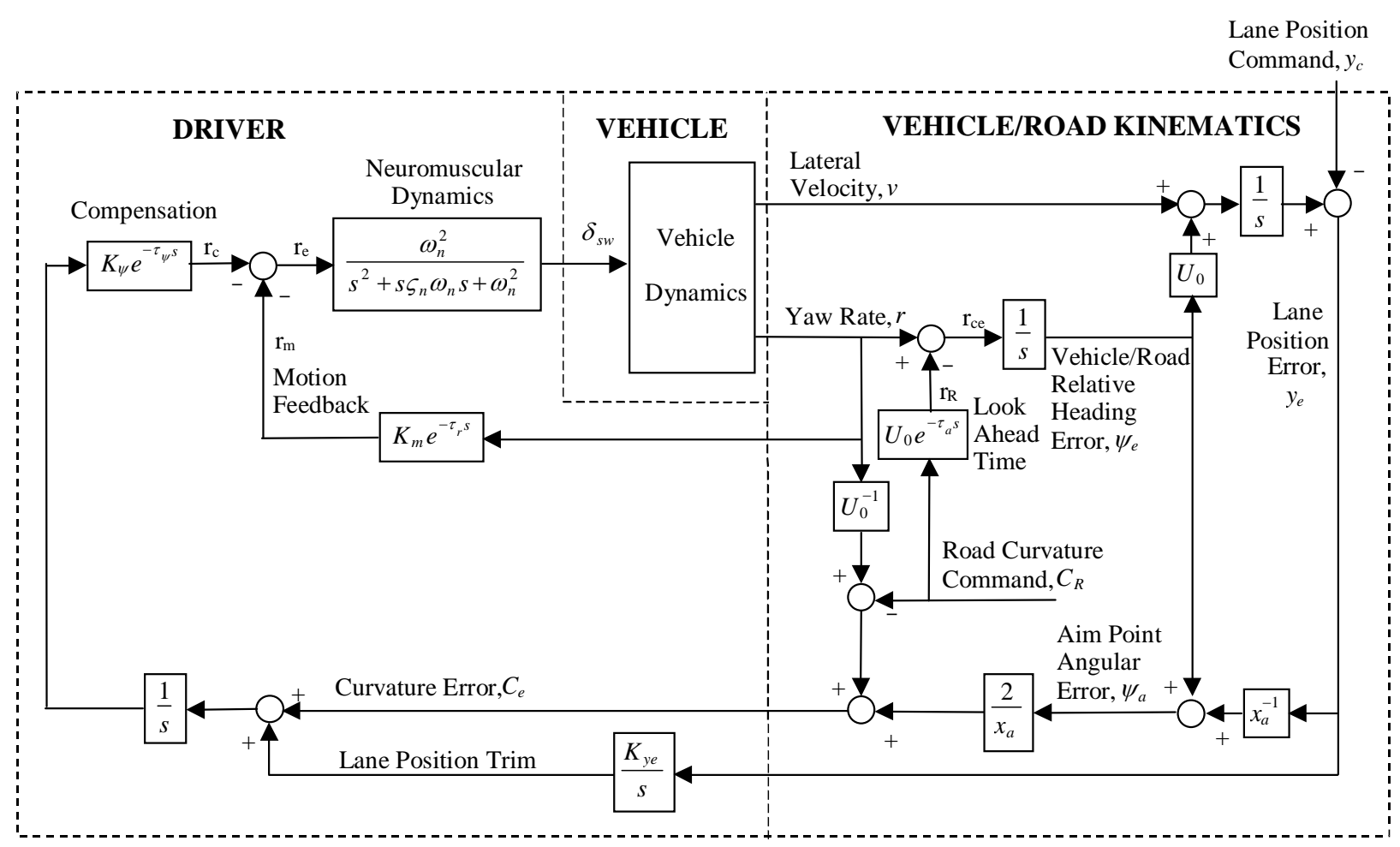

Fig. 11 Block diagram of the driver model

The fifteen maneuvers used in this paper are summarized in Table 2. The maneuvers used in training the Neural Network (NN) are marked with “*” in the scenario column. The rest are used as evaluation maneuvers to assess the performance of the trained NN. 
Table 2 Summary of the fifteen maneuvers

\begin{tabular}{|c|c|c|c|c|}
\hline Pattern & Scenario & $\begin{array}{l}\text { Speed } \\
\text { (mph) }\end{array}$ & Description & Rollover \\
\hline \multirow{4}{*}{$\begin{array}{l}\text { Ramp } \\
\text { steering }\end{array}$} & R1 & 40 & $180 \mathrm{deg}$ in $10 \mathrm{sec}$ & Yes \\
\hline & R2 & 60 & $180 \mathrm{deg}$ in $10 \mathrm{sec}$ & Yes \\
\hline & $\mathrm{R} 3 *$ & 40 & 180 deg in $20 \mathrm{sec}$ & Yes \\
\hline & $\mathrm{R} 4 *$ & 60 & 180 deg in $20 \mathrm{sec}$ & Yes \\
\hline \multirow{4}{*}{$\begin{array}{l}\text { Entering } \\
\text { ramp }\end{array}$} & E1* & 25 & Radius $=200 \mathrm{ft}$ & No \\
\hline & E2 & 40 & Radius $=200 \mathrm{ft}$ & Yes \\
\hline & $\mathrm{E} 3 *$ & 40 & Radius $=500 \mathrm{ft}$ & No \\
\hline & E4 & 60 & Radius $=500 \mathrm{ft}$ & Yes \\
\hline \multirow[t]{5}{*}{$\begin{array}{l}\text { Obstacle } \\
\text { avoidance }\end{array}$} & O1 & 40 & $\begin{array}{l}\text { Obstacle } 1 \mathrm{sec} \\
\text { ahead }\end{array}$ & No \\
\hline & $\mathrm{O} 2 *$ & 40 & 2 sec ahead & No \\
\hline & O3* & 60 & 1 sec ahead & Yes \\
\hline & $\mathrm{O} 4$ & 60 & 3 sec ahead & No \\
\hline & O5 & 70 & $1.5 \mathrm{sec}$ ahead & Yes \\
\hline \multirow[t]{2}{*}{ Worst-case } & W1* & 60 & 130 deg limit & Yes \\
\hline & W2 & 70 & 105 degree limit & Yes \\
\hline
\end{tabular}

Note: *Training maneuvers

\section{SIMULATION RESULTS}

\subsection{TTR and ArcSim TTR}

Since excessive roll motion usually starts from the semi-trailer unit, we chose to monitor the motion of the semi-trailer. The critical roll angle of the semi-trailer was selected as 5.6 deg from a step steering input with a tire near-lift-off condition. In our implementations, TTR is saturated at 3 sec (see Fig. 12). As mentioned in previous sections, TTR can be calculated from either a full nonlinear complex or a linear simple model. Here the simple decoupled yaw-roll model is used for simple TTR calculation. For comparison purposes, we also defined an “ArcSim TTR,” which was obtained from the ArcSim model instead of the simple model.

Figure 12 shows the comparison between the simple TTR (from the simple model) and the ArcSim TTR for four representative maneuvers. It can be seen that due to the difference between the two models, the simple TTR can be very different from (worse than) the ArcSim TTR. The simple TTR tends to be conservative, i.e., rollovers are predicted to occur sooner than they really do. Another interesting thing to notice is that the slope of the ArcSim TTR always approaches -1 (the desired TTR) when rollover occurs. Figure 12 also shows that the simple model is not accurate enough to generate a true "count-down” toward rollover across the all types of maneuvers. Obviously, a more innovative technique is required to improve the performance of the simple model. 

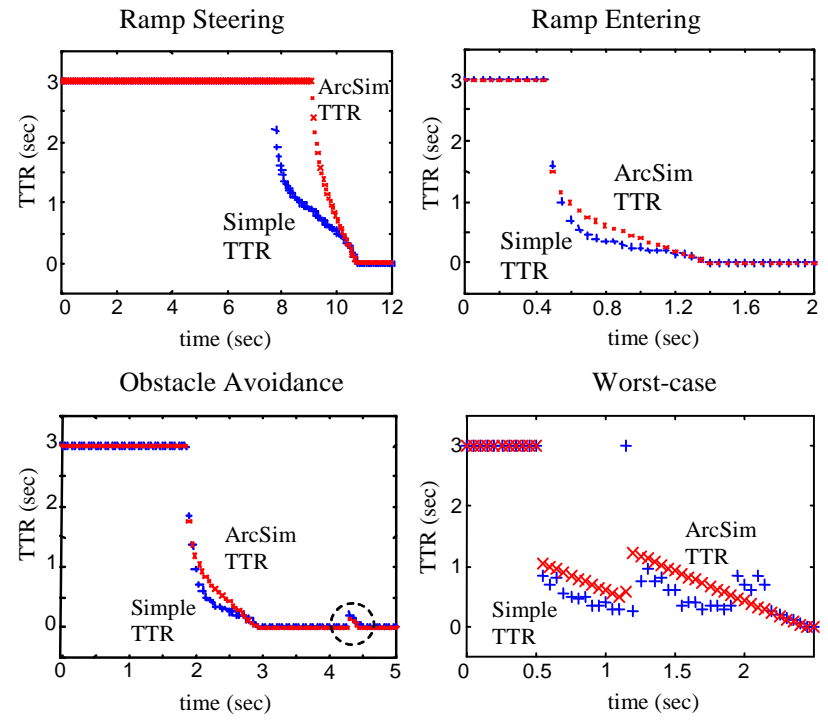

Fig. 12 Simple TTR and ArcSim TTR

The representative ArcSim responses under an obstacle avoidance (shown in Fig. 12) maneuver are shown in Fig. 13. The responses agreed with the TTR results. After the first star mark, the roll angle of the semi-trailer exceeded 5.6 deg. The TTR stopped counting and remained zero. The second set of nonzero TTR points (marked with a circle in Fig. 12) was the result of reversing roll dynamics. The roll angle of the trailer was changing from 5.6 deg to -5.6 deg. After the second star mark, the roll angle dropped below $5.6 \mathrm{deg}$. Therefore, the TTR began counting the rollover threat toward -5.6 deg. After the third star mark, the truck actually rolled over.
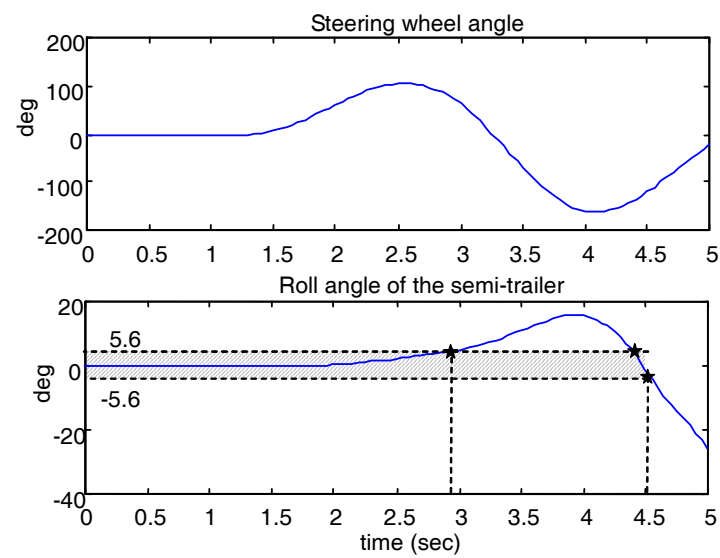

Fig. 13 ArcSim responses under the obstacle avoidance maneuver

\section{$\underline{5.2 \text { Neural Network TTR }}$}

From the TTR results shown in the previous subsection, it is obvious that the simple TTR is not accurate enough. Ideally, an accurate TTR should count down toward rollover incidents under all driving scenarios. In this 
case study, we limited the scope of driving scenarios to the fifteen cases shown in Table 2. The purpose is to produce a new TTR that uniformly counts down toward rollover incidents for all the fifteen scenarios.

A NN was constructed to generate the NN-TTR (see Fig. 3). The inputs of the NN are simple TTR, roll angle, and change of the roll angle of the semi-trailer. The desired output from the NN is a straight line of slope $=-1$ for all the training scenarios involving "rollovers" illustrated in Table 2. For non-rollover training scenarios, the desired output of the NN is a straight line of slope $=0$ (i.e. NN-TTR $=3 \mathrm{sec}$ for the simulated time horizon). The purpose of including non-rollover training scenarios is to prevent false alarms (false positive readings).

The NN has two hidden layers with 9 neurons and 1 neuron for the layers 1 and 2, respectively. The functions of the neurons are standard tansig functions. The NN was first trained by using 6 training scenarios listed in Table 2 (without the worst-case training scenario). The training stopped when a pre-set performance threshold was satisfied. Remaining evaluation scenarios (shown in Table 2) were then used to validate the performance of the NN. These maneuvers can be grouped into three categories (see Fig. 4) according to the time at which a rollover develops: Mild (ramp steering), Bad (ramp entering and obstacle avoidance), and Worst-Case. Statistical results are reported in Table 3 for these three categories, and representative results for the four driving patterns are shown in Fig. 14.

Table 3 Statistical NN-TTR results (without worst-case training scenario)

\begin{tabular}{|l|c|c|c|}
\hline NN-TTR error & Mild & Bad & Worst-Case \\
\hline Mean (sec) & 0.004 & 0.032 & 0.110 \\
\hline Standard deviation (sec) & 0.011 & 0.074 & 0.025 \\
\hline
\end{tabular}
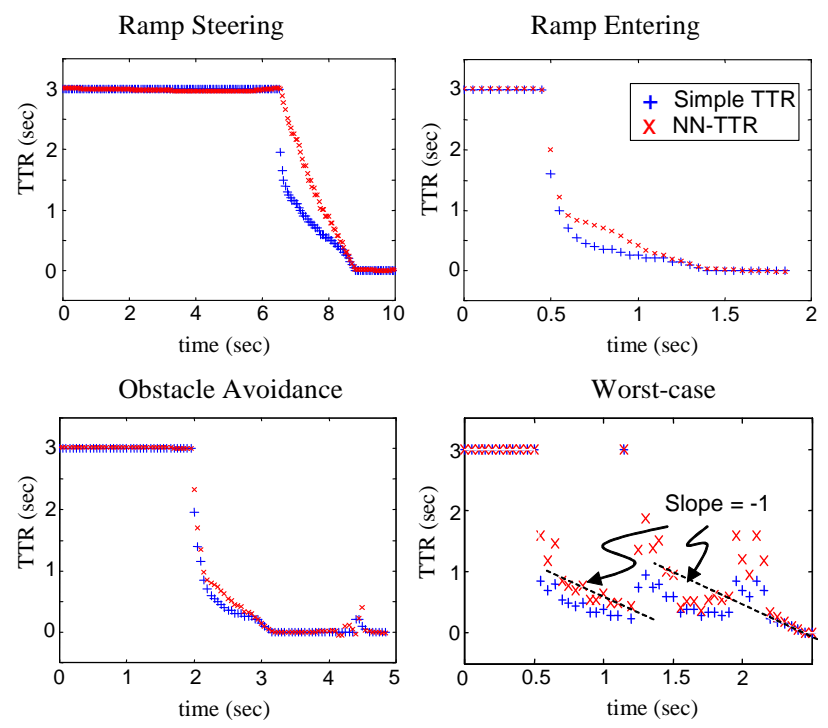

Fig. 14 NN-TTR results without worst-case training scenario 
It can be seen that the NN-TTR indeed approximates a straight line of slope -1 (desired TTR) in all the maneuvers except for the worst-case. The performance deteriorates progressively as the steering excitation becomes too violent. Because the rollover occurs most unexpectedly for the "worst-case," the NN-TTR values were widely scattered around the line of the slope -1 and the performance of the NN-TTR was the worst. Fortunately, the "worst-case" maneuver was obtained by searching through the steering space and should not occur frequently in real-life. It nevertheless gives us an estimation of the worst possible performance one can expect from this NNTTR. After all 7 training scenarios (including the worst-case) were used in the NN training, the new results are shown in Table 4 and Fig. 15.

Table 4 Statistical NN-TTR results (with worst-case training scenario)

\begin{tabular}{|l|c|c|c|}
\hline NN-TTR error & Mild & Bad & Worst-Case \\
\hline Mean (sec) & 0.005 & 0.022 & 0.030 \\
\hline Standard deviation (sec) & 0.015 & 0.034 & 0.060 \\
\hline
\end{tabular}

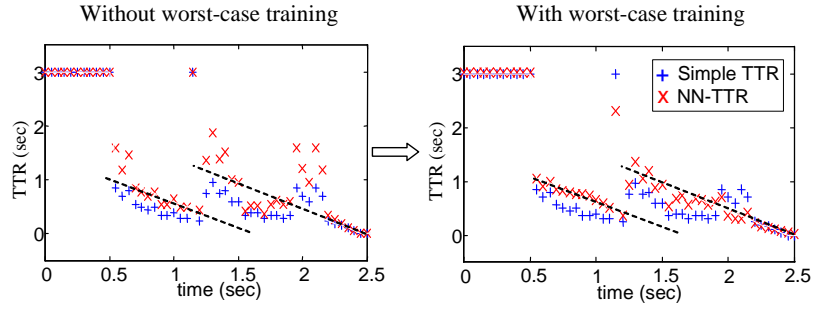

Fig. 15 NN-TTR results with and without worst-case training scenario

As can be seen from Fig. 15, the NN-TTR values with the worst-case training scenario were less scattered around the line of slope -1 than the values without the worst-case training scenario. Although Table 4 shows that the performance of the NN-TTR for the mild categories is deteriorated slightly, the performances for the other two categories are improved significantly with worst-case maneuver included in the training set. Based on the fact that the standard deviation under typical (bad) rollover patterns is only $34 \mathrm{~ms}$, and that under the worst-case is $60 \mathrm{~ms}$, the performance of the proposed NN-TTR is determined to be satisfactory.

\section{CONCLUSIONS}

A Time-To-Rollover (TTR) metric was developed in this paper to assess the rollover threat for an articulated vehicle. A simple decoupled yaw-roll model, which is suitable for faster-than-real-time implementation, was developed by using system identification techniques. The yaw model is a 4-state bicycle-towing-unicycle model, 
and the roll model is an 8-state model that includes separate sprung mass and unsprung mass roll degrees of freedom for the tractor and trailer units, respectively. This simple model was found to be much faster than real-time on a Pentium class computer and thus can be used for real-time TTR computations. However, the TTR obtained from the simple model (simple TTR) did not accurately reflect rollover threats due to its simplicity. A Neural Network (NN) was then added and trained to generate a uniform TTR (NN-TTR) countdown for an array of test maneuvers, which covered a wide spectrum from very smooth to very violent rollover incidents. The NN takes simple TTR, vehicle roll angle, and change of roll angle as inputs, and produces an output in the shape of a straight line with slope $=-1$ (the desired TTR). The standard deviation of NN-TTR was found to be about $30 \mathrm{~ms}$ for rollover maneuvers commonly seen on highways and $60 \mathrm{~ms}$ for the identified worst-case scenario. By including the worst-case maneuvers to complete the NN training, the overall performance of NN-TTR can be improved across the test maneuvers considered in this paper.

\section{ACKNOWLEDGMENTS}

This research was supported by the U.S. Army TARDEC under the contract DAAE07-94-C-R094.

\section{REFERENCES}

1. Blower, D. F., 1997, “Truck and Bus Crash Factbook 1995," UMTRI 97-30, University of Michigan Transportation Research Institute, Center for National Truck Statistics, Ann Arbor, MI.

2. Woodrooffe, J. H. F., 1993, "Practical Concepts in Heavy Truck Rollover Accident Analysis,” Heavy Vehicle Rollovers - A Safety Issue TOPTEC, Society of Automotive Engineers, Warrendale, Pa.

3. Rakheja, S., and Pichè, A., 1990, "Development of Directional Stability Criteria for an Early Warning Safety Device,” SAE Paper No. 902265.

4. Preston-Thomas, J., and Woodrooffe, J. H. F., 1990, “A Feasibility Study of a Rollover Warning Device for Heavy Trucks,” TP 10610E, Transport Canada.

5. Freedman, M., Olson, P. L., and Zador, P. L., 1992, “Speed Actuated Rollover Advisory Signs for Trucks on Highway Exit Ramps,” Insurance Institute for Highway Safety and University of Michigan Transportation Research Institute. 
6. McGee, H., Joshua, S., Hughes, W., Strickland, R., Bareket, Z., and Fancher, P., 1993, "Feasibility of An Automatic Truck Warning System,” FHWA-RD-93-039, U.S. Department of Transportation, Federal Highway Administration, Research and Development, Turner-Fairbank Highway Research Center, McLean, VA.

7. Strickland, R., and McGee, H., 1998 "Evaluation of Prototype Automatic Truck Rollover Warning Systems," FHWA-RD-93-039, U.S. Department of Transportation, Federal Highway Administration, Research and Development, Turner-Fairbank Highway Research Center, McLean, VA.

8. Ervin, R., Winkler, C., Fancher, P., Hagan, M., Krishnaswami, V., Zhang, H., Bogard, S., Karamihas, S., 1998, "Cooperative Agreement to Foster the Deployment of a Heavy Vehicle Intelligent Dynamic Stability Enhancement System,” UMTRI-98-3, University of Michigan Transportation Research Institute, Ann Arbor, MI.

9. Nalecz, A. G., and Bindemann, A. C., 1987, "Sensitivity Analysis of Vehicle Design Attributes that Affect Vehicle Response in Critical Accident Situations - Part I: User's Manual,” DOT-HS-807-229, U.S. Department of Transportation, National Highway Transportation Safety Administration.

10. Nalecz, A. G., 1991, "Intermediate Maneuver Induced Rollover Simulation (IMIRS) a Sensitivity Analysis," DOT-HS-807-672, U.S. Department of Transportation, National Highway Transportation Safety Administration.

11. Nalecz, A. G., Lu, Z., and d'Entremont, K. L., 1993, “An investigation into Dynamic Measure of Vehicle Rollover Propensity,” SAE Paper No. 930831.

12. Hayward, J. C., 1972, "Near Miss Determination through Use of A Scale of Danger," Highway Research Record, No. 384, pp. 24-34.

13. Lin, C.-F., 1995, "Lane sensing and path prediction for preventing vehicle road departure accidents," Ph.D. dissertation, University of Michigan, Ann Arbor, MI.

14. Lin, C.-F., and Ulsoy, A. G., 1996, "Time to lane crossing calculation and characterization of its uncertainty," ITS Journal, 3(2), pp. 85-98.

15. Lin, C.-F., Ulsoy, A. G., and LeBlanc, D. J., 1999, "Lane geometry perception and the characterization of its associated uncertainty,” Journal of Dynamic Systems, Measurement and Control, Transactions of the ASME, 121(6), pp. 1-9.

16. Lin, C.-F., Ulsoy, A. G., and LeBlanc, D. J., 2000, "Vehicle Dynamics and External Disturbance Estimation for Vehicle Path Prediction,” IEEE Transactions on Control Systems Technology, 8(3), pp. 508-518. 
17. Ma, W. and Peng, H., 1996, "Worst-case evaluation methods for vehicle control systems," Proceedings of the 1996 ASME International Mechanical Engineering Congress and Exposition.

18. Ma, W. and Peng, H., 1999, “Worst-case evaluation method for dynamic systems,” Journal of Dynamic Systems, Measurement and Control, Transactions of the ASME, 121(2), pp. 191-199.

19. Allen, R. W., Rosenthal, T. J., and Szostak, H. T., 1988, "Analytical Modeling of Driver Response in Crash Avoidance Maneuvering, Volume I: Technical Background," DOT-HS-807-270, U.S. Department of Transportation, National Highway Transportation Safety Administration.

20. Weir, D.H. and McRuer, D.T., 1968, "A Theory of for Driver Steering Control of Motor Vehicles," Highway Research Record, No. 247, pp.7-28.

\section{Appendix A}

Detail equations of the four simple roll models are shown in this appendix. Small angle approximations, $\sin \phi \cong \phi, \cos \phi \cong 1$, and $\phi^{2} \cong 0$, are applied for all roll angles through the derivation.

Nomenclature:

W Vehicle total weight

$a_{y} \quad$ Lateral acceleration (unit: g)

$T \quad$ Half of the vehicle track width

$h \quad$ C.G. height

$K_{t} \quad$ Tire stiffness

$\phi_{s} \quad$ Sprung mass roll angle w.r.t. the axle

$\phi_{u} \quad$ Unsprung mass roll angle

$\phi \quad$ Sprung mass roll angle, $\left(\phi=\phi_{u}+\phi_{s}\right)$

$F_{z} \quad$ Axle load

$W_{u} \quad$ Weight of the unsprung mass

$W_{s} \quad$ Weight of the sprung mass

$h_{r} \quad$ Height of roll center 
$h_{s} \quad$ Sprung mass C.G. height

$R_{t} \quad$ Unsprung mass C.G. height

$K_{\mathrm{s}} \quad$ Spring stiffness-per side

$K_{s a} \quad$ Auxiliary roll stiffness

$S \quad$ Distance between springs

$K_{\phi s} \quad$ Roll stiffness of an axle, $K_{\phi s}=1 / 2 \cdot S^{2} \cdot K_{s}+K_{s a}$

Model A (one unit, no axle)

It this model, the tractor and trailer are lumped into one unit. Furthermore, we do not distinguish the axles from the sprung mass. The tires and suspension springs are also assumed to be infinitely stiff. It is easy to show that the axle of a rigid vehicle will lift-off at $\phi=0$ and $a_{y}=T / h$.

Model B (one unit, one axle, one DOF)

For this model, we assume that the tire stiffness is considered. The axle and the sprung mass, however, are connected together by a rigid suspension. From the roll moment balance around the mass center, we have

$$
W \cdot a_{y} \cdot h+\left(\frac{W}{2}-K_{t} \cdot \phi \cdot T\right) \cdot(T+h \cdot \phi)=\left(\frac{W}{2}+K_{t} \cdot \phi \cdot T\right) \cdot(T-h \cdot \phi)
$$

At axle lift-off, normal force of the inner tire becomes zero, i.e. $\frac{W}{2}-K_{t} \cdot \phi \cdot T=0$. Therefore, the critical roll and acceleration values are $\phi=\frac{W}{2 K_{t} \cdot T}$ and $a_{y}=\frac{T}{h}-\frac{W}{2 K_{t} \cdot T}$, respectively.

Model C (one unit, one axle, two DOF)

Model C is obtained from Model B by adding a flexible suspension which separates the sprung and the unsprung mass. From the roll moment balance about the unsprung mass C.G., we have

$$
\begin{aligned}
& K_{\phi s} \cdot \phi_{s}+F_{u} \cdot\left(h_{r}-R_{t}\right) \cdot \phi_{u}+F_{u} \cdot a_{y} \cdot\left(h_{r}-R_{t}\right)+F_{z} \cdot a_{y} \cdot R_{t}+\left(\frac{F_{z}}{2}-K_{t} \cdot \phi_{u} \cdot T\right) \cdot\left(T+R_{t} \cdot \phi_{u}\right) \\
&=\left(\frac{F_{z}}{2}+K_{t} \cdot \phi_{u} \cdot T\right) \cdot\left(T-R_{t} \cdot \phi_{u}\right)
\end{aligned}
$$

or, 


$$
\left[F_{u} \cdot\left(h_{r}-R_{t}\right)+F_{z} \cdot R_{t}\right] \cdot a_{y}+K_{\phi s} \cdot \phi_{S}+\left[F_{u} \cdot\left(h_{r}-R_{t}\right)-2 K_{t} \cdot T^{2}+F_{z} \cdot R_{t}\right] \cdot \phi_{u}=0
$$

Similarly, take roll moment balance for the sprung mass C.G., we can get

$$
F_{u} \cdot\left(h_{s}-h_{r}\right) \cdot \phi+F_{u} \cdot a_{y} \cdot\left(h_{s}-h_{r}\right)=K_{\phi s} \cdot \phi_{s}
$$

Finally, at axle lift-off, we have $\frac{F_{z}}{2}-K_{t} \cdot \phi_{u} \cdot T=0$, i.e.,

$$
\phi_{u}=\frac{F_{z}}{2 K_{t} \cdot T}
$$

From Eq. (A.1) to Eq. (A.3), plus the definition of $\phi=\phi_{u}+\phi_{s}$, we have 4 equations and 4 unknowns ( $a_{y}, \phi_{u}$, $\phi_{s}$, and $\phi$ ). Therefore, the roll and lateral acceleration threshold values can be obtained by solving above equations.

Model D (two units, sprung and unsprung mass for each unit, four DOF)

Take roll moment balance around the tractor unsprung mass C.G., we have

$$
\begin{array}{r}
K_{\phi s 1} \cdot \phi_{s 1}+F_{u 1} \cdot\left(h_{r 1}-R_{t 1}\right) \cdot \phi_{u 1}+F_{u 1} \cdot a_{y} \cdot\left(h_{r 1}-R_{t 1}\right)+F_{z 1} \cdot a_{y} \cdot R_{t 1}+\left(\frac{F_{z 1}}{2}-K_{t 1} \cdot \phi_{u 1} \cdot T_{1}\right) \cdot\left(T_{1}+R_{t 1} \cdot \phi_{u 1}\right) \\
=\left(\frac{F_{z 1}}{2}+K_{t 1} \cdot \phi_{u 1} \cdot T_{1}\right) \cdot\left(T_{1}-R_{t 1} \cdot \phi_{u 1}\right)
\end{array}
$$

or,

$$
\left[F_{u 1} \cdot\left(h_{r 1}-R_{t 1}\right)+F_{z 1} \cdot R_{t 1}\right] \cdot a_{y}+K_{\phi s 1} \cdot \phi_{s 1}+\left[F_{u 1} \cdot\left(h_{r 1}-R_{t 1}\right)-2 K_{t 1} \cdot T_{1}^{2}+F_{z 1} \cdot R_{t 1}\right] \cdot \phi_{u 1}=0
$$

Similarly, moment balance around the trailer unsprung mass C.G. results in

$$
\begin{array}{r}
K_{\phi s 2} \cdot \phi_{s 2}+F_{u 2} \cdot\left(h_{r 2}-R_{t 2}\right) \cdot \phi_{u 2}+F_{u 2} \cdot a_{y} \cdot\left(h_{r 2}-R_{t 2}\right)+F_{z 2} \cdot a_{y} \cdot R_{t 2}+\left(\frac{F_{z 2}}{2}-K_{t 2} \cdot \phi_{u 2} \cdot T_{2}\right) \cdot\left(T_{2}+R_{t 2} \cdot \phi_{u 2}\right) \\
=\left(\frac{F_{z 2}}{2}+K_{t 2} \cdot \phi_{u 2} \cdot T_{2}\right) \cdot\left(T_{2}-R_{t 2} \cdot \phi_{u 2}\right)
\end{array}
$$

or,

$$
\left[F_{u 2} \cdot\left(h_{r 2}-R_{t 2}\right)+F_{z 2} \cdot R_{t 2}\right] \cdot a_{y}+K_{\phi s 2} \cdot \phi_{s 2}+\left[F_{u 2} \cdot\left(h_{r 2}-R_{t 2}\right)-2 K_{t 2} \cdot T_{2}^{2}+F_{z 2} \cdot R_{t 2}\right] \cdot \phi_{u 2}=0
$$

Axle lift-off occurs when the normal force on the trailer axle becomes zero. Therefore, the following condition is satisfied at axle lift-off: 


$$
\phi_{u 2}=\frac{F_{z 2}}{2 K_{t 2} \cdot T_{2}}
$$

Moment balance around the sprung masses of the tractor and trailer results in

$$
F_{u 1} \cdot\left(h_{s 1}-h_{r 1}\right) \cdot a_{y}+\left(h_{5}-h_{s 1}\right) \cdot F_{5 y}-K_{\phi s 1} \cdot \phi_{s 1}+\left[F_{5 z} \cdot\left(h_{5}-h_{s 1}\right)+F_{u 1} \cdot\left(h_{s 1}-h_{r 1}\right)-K_{5}\right] \cdot \phi_{1}+K_{5} \cdot \phi_{2}=0
$$

and

$$
F_{u 2} \cdot\left(h_{s 2}-h_{r 2}\right) \cdot a_{y}+\left(h_{s 2}-h_{5}\right) \cdot F_{5 y}-K_{\phi s 2} \cdot \phi_{s 2}+K_{5} \cdot \phi_{1}+\left[F_{5 z} \cdot\left(h_{s 2}-h_{5}\right)+F_{u 2} \cdot\left(h_{s 2}-h_{r 2}\right)-K_{5}\right] \cdot \phi_{2}=0
$$

respectively. At steady-state cornering, the lateral force at the fifth wheel has to satisfy the relationship $F_{5 y}+W_{s 1} \cdot a_{y}=F_{u 1} \cdot a_{y}$. After simplification, we can obtain

$$
F_{5 y}=F_{5 z} \cdot a_{y}
$$

From Eq. (A.4) to Eq. (A.9), plus the definitions of $\phi_{1}=\phi_{u 1}+\phi_{s 1}$ and $\phi_{2}=\phi_{u 2}+\phi_{s 2}$, we have 8 equations and 8 unknowns $\left(a_{y}, F_{y}, \phi_{u 1}, \phi_{u 2}, \phi_{s 1}, \phi_{s 2}, \phi_{1}, \phi_{2}\right)$. Therefore, the roll and lateral acceleration threshold values of the sprung masses of the tractor and trailer can be obtained respectively by solving above equations.

\section{$\underline{\text { Dynamic Model D }}$}

Since Model D was found to be accurately enough to capture the steady-state turning behavior of ArcSim, we can construct a dynamic version of Model D to predict the roll motions of ArcSim. In the dynamic model, the time derivatives of the roll angles are no longer assumed to be zero. The damping and inertia terms thus need to be defined and included in the dynamic equations. The free body diagram (FBD) of the upsprung mass and the sprung mass of the tractor are shown in Fig. A.1. and Fig. A.2, respectively. Additional Nomenclature for the dynamic model are defined as follows:

D Distance between shock absorbers

$B_{s} \quad$ Shock absorber rate

$B_{\phi s} \quad$ Roll damping coefficent at an axle, $B_{\phi s}=1 / 2 \cdot D^{2} \cdot B_{s}$

$I_{x x u} \quad$ Moment of inertia of the unsprung mass

$I_{x x s} \quad$ Moment of inertia of the sprung mass 


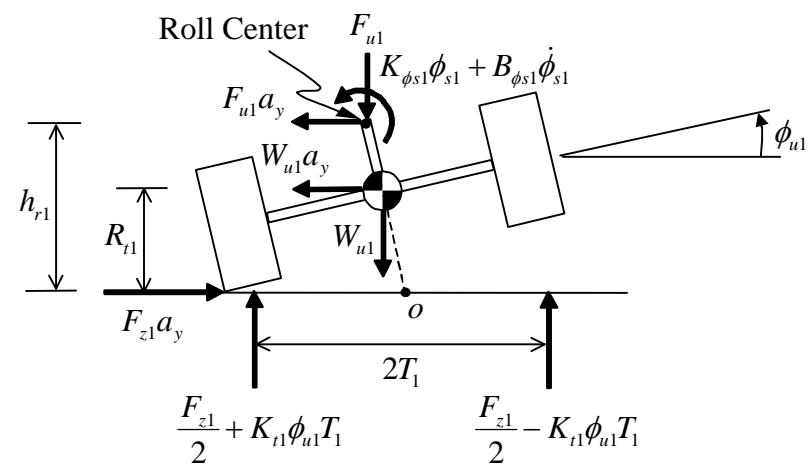

Fig. A.1 Free body diagram of the tractor unsprung mass

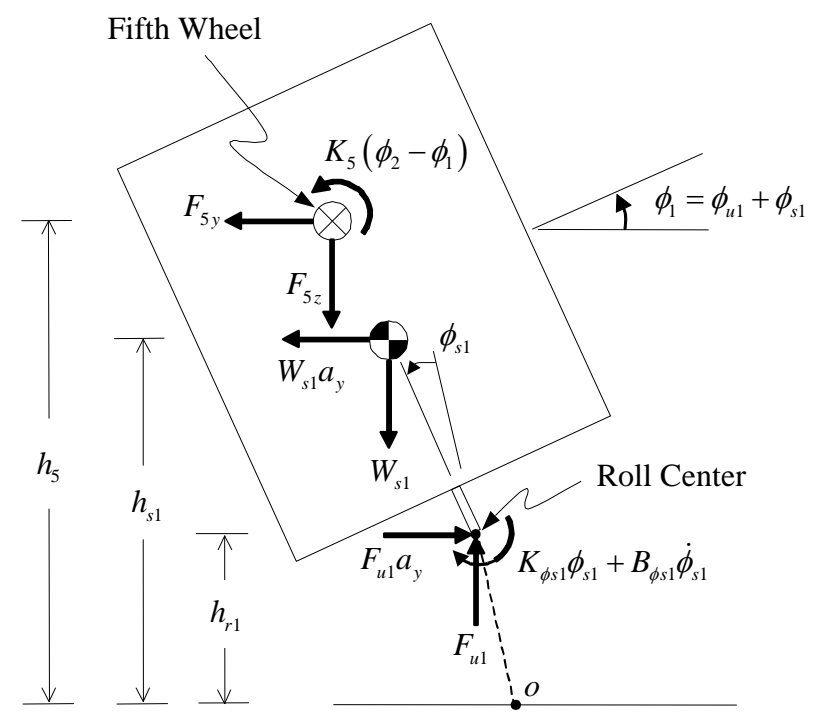

Fig. A.2 Free body diagram of the tractor sprung mass

From the moment balance around the unsprung mass center of the tractor, we obtain

$$
\begin{aligned}
\ddot{\phi}_{u 1}= & \frac{1}{I_{x x u 1}} \cdot\left\{\left[F_{u 1} \cdot\left(h_{r 1}-R_{t 1}\right)-2 K_{t 1} \cdot T_{1}^{2}-K_{\phi s 1}+F_{z 1} \cdot R_{t 1}\right] \cdot \phi_{u 1}-B_{\phi s 1} \cdot \dot{\phi}_{u 1}+K_{\phi s 1} \cdot \phi_{1}+B_{\phi s 1} \cdot \dot{\phi}_{1}\right\} \\
& +\frac{1}{I_{x x u 1}}\left[F_{u 1} \cdot\left(h_{r 1}-R_{t 1}\right)+F_{z 1} \cdot R_{t 1}\right] \cdot a_{y}
\end{aligned}
$$

The FBD of the trailer unsprung mass is identical to that of the tractor unsprung mass, except that the subscript of all the variables need to be changed from "1" (tractor) to "2" (trailer). The dynamic equation can then be obtained as follows:

$$
\ddot{\phi}_{u 2}=\frac{1}{I_{x x u 2}} \cdot\left\{\left[F_{u 2} \cdot\left(h_{r 2}-R_{t 2}\right)-2 K_{t 2} \cdot T_{2}^{2}-K_{\phi s 2}+F_{z 2} \cdot R_{t 2}\right] \cdot \phi_{u 2}-B_{\phi s 2} \cdot \dot{\phi}_{u 2}+K_{\phi s 2} \cdot \phi_{2}+B_{\phi s 2} \cdot \dot{\phi}_{2}\right\}
$$




$$
+\frac{1}{I_{x x u 2}}\left[F_{u 2} \cdot\left(h_{r 2}-R_{t 2}\right)+F_{z 2} \cdot R_{t 2}\right] \cdot a_{y}
$$

The dynamic equation for the sprung mass of the tractor is

$$
\begin{aligned}
\ddot{\phi}_{1}= & \frac{1}{I_{x x s 1}} \cdot\left\{K_{\phi s 1} \cdot \phi_{u 1}+B_{\phi s 1} \cdot \dot{\phi}_{u 1}+\left[F_{5 z} \cdot\left(h_{5}-h_{s 1}\right)+F_{u 1} \cdot\left(h_{s 1}-h_{r 1}\right)-K_{5}-K_{\phi s 1}\right] \cdot \phi_{1}-B_{\phi s 1} \cdot \dot{\phi}_{1}+K_{5} \cdot \phi_{2}\right\} \\
& +\frac{1}{I_{x x s 1}} \cdot\left[F_{5 z} \cdot\left(h_{5}-h_{s 1}\right)+F_{u 1} \cdot\left(h_{s 1}-h_{r 1}\right)\right] \cdot a_{y}
\end{aligned}
$$

The FBD shown in Fig. A.2 can also be applied to derive the dynamic equation of the trailer sprung mass. Again, the only difference is that the subscript of all the variables need to be changed from "1" (tractor) to "2" (trailer). The dynamic equation for the trailer sprung mass is thus:

$$
\begin{aligned}
\ddot{\phi}_{2}= & \frac{1}{I_{x x s 2}} \cdot\left\{K_{\phi s 2} \cdot \phi_{u 2}+B_{\phi s 2} \cdot \dot{\phi}_{u 2}+K_{5} \cdot \phi_{1}+\left[F_{5 z} \cdot\left(h_{s 2}-h_{5}\right)+F_{u 2} \cdot\left(h_{s 2}-h_{r 2}\right)-K_{\phi s 2}-K_{5}\right] \cdot \phi_{2}-B_{\phi s 2} \cdot \dot{\phi}_{2}\right\} \\
& +\frac{1}{I_{x x s 2}} \cdot\left[F_{5 z} \cdot\left(h_{s 2}-h_{5}\right)+F_{u 2} \cdot\left(h_{s 2}-h_{r 2}\right)\right] \cdot a_{y}
\end{aligned}
$$

Eq. (A.10), (A.11), (A.12), and (A.13) are four second order differential equations describing the roll plane dynamics of ArcSim. They can be put into a standard state equation form $\dot{x}=A \cdot x+b \cdot a_{y}$. The state vector $x=\left[\begin{array}{llllllll}\phi_{u 1} & \dot{\phi}_{u 1} & \phi_{u 2} & \dot{\phi}_{u 2} & \phi_{1} & \dot{\phi}_{1} & \phi_{2} & \dot{\phi}_{2}\end{array}\right]^{T}$, and

$$
A=\left[\begin{array}{cccccccc}
0 & 1 & 0 & 0 & 0 & 0 & 0 & 0 \\
a_{21} & a_{22} & 0 & 0 & a_{25} & a_{26} & 0 & 0 \\
0 & 0 & 0 & 1 & 0 & 0 & 0 & 0 \\
0 & 0 & a_{43} & a_{44} & 0 & 0 & a_{47} & a_{48} \\
0 & 0 & 0 & 0 & 0 & 1 & 0 & 0 \\
a_{61} & a_{62} & 0 & 0 & a_{65} & a_{66} & a_{67} & 0 \\
0 & 0 & 0 & 0 & 0 & 0 & 0 & 1 \\
0 & 0 & a_{83} & a_{84} & a_{85} & 0 & a_{87} & a_{88}
\end{array}\right], b=\left[\begin{array}{c}
0 \\
b_{2} \\
0 \\
b_{4} \\
0 \\
b_{6} \\
0 \\
b_{8}
\end{array}\right]
$$

where

$$
\begin{aligned}
& a_{21}=\frac{1}{I_{x x u 1}} \cdot\left[F_{u 1} \cdot\left(h_{r 1}-R_{t 1}\right)-2 K_{t 1} \cdot T_{1}^{2}-K_{\phi s 1}+F_{z 1} \cdot R_{t 1}\right], a_{22}=\frac{1}{I_{x x u 1}} \cdot\left(-B_{\phi s 1}\right), a_{25}=\frac{1}{I_{x x u 1}} \cdot K_{\phi s 1}, \\
& a_{26}=\frac{1}{I_{x x u 1}} \cdot B_{\phi s 1}, a_{43}=\frac{1}{I_{x x u 2}} \cdot\left[F_{u 2} \cdot\left(h_{r 2}-R_{t 2}\right)-2 K_{t 2} \cdot T_{2}^{2}-K_{\phi s 2}+F_{z 2} \cdot R_{t 2}\right], a_{44}=\frac{1}{I_{x x u 2}} \cdot\left(-B_{\phi s 2}\right),
\end{aligned}
$$




$$
\begin{aligned}
& a_{47}=\frac{1}{I_{x x u 2}} \cdot K_{\phi s 2}, a_{48}=\frac{1}{I_{x x u 2}} \cdot B_{\phi s 2}, a_{61}=\frac{1}{I_{x x s 1}} \cdot K_{\phi s 1}, a_{62}=\frac{1}{I_{x x s 1}} \cdot B_{\phi s 1}, \\
& a_{65}=\frac{1}{I_{x x s 1}} \cdot\left[F_{5 z} \cdot\left(h_{5}-h_{s 1}\right)+F_{u 1} \cdot\left(h_{s 1}-h_{r 1}\right)-K_{5}-K_{\phi s 1}\right], a_{66}=\frac{1}{I_{x x s 1}} \cdot\left(-B_{\phi s 1}\right), a_{67}=\frac{1}{I_{x x s 1}} \cdot K_{5}, \\
& a_{83}=\frac{1}{I_{x x s 2}} \cdot K_{\phi s 2}, a_{84}=\frac{1}{I_{x x s 2}} \cdot B_{\phi s 2}, a_{85}=\frac{1}{I_{x x s 2}} \cdot K_{5}, \\
& a_{87}=\frac{1}{I_{x x s 2}} \cdot\left[F_{5 z} \cdot\left(h_{s 2}-h_{5}\right)+F_{u 2} \cdot\left(h_{s 2}-h_{r 2}\right)-K_{\phi s 2}-K_{5}\right], a_{88}=\frac{1}{I_{x x s 2}} \cdot\left(-B_{\phi s 2}\right), \\
& b_{2}=\frac{1}{I_{x x u 1}} \cdot\left[F_{u 1} \cdot\left(h_{r 1}-R_{t 1}\right)+F_{z 1} \cdot R_{t 1}\right], b_{4}=\frac{1}{I_{x x u 2}} \cdot\left[F_{u 2} \cdot\left(h_{r 2}-R_{t 2}\right)+F_{z 2} \cdot R_{t 2}\right], \\
& b_{6}=\frac{1}{I_{x x s 1}} \cdot\left[F_{5 z} \cdot\left(h_{5}-h_{s 1}\right)+F_{u 1} \cdot\left(h_{s 1}-h_{r 1}\right)\right], b_{8}=\frac{1}{I_{x x s 2}} \cdot\left[F_{5 z} \cdot\left(h_{s 2}-h_{5}\right)+F_{u 2} \cdot\left(h_{s 2}-h_{r 2}\right)\right] .
\end{aligned}
$$

Notice that the input to this state equation is the lateral acceleration $a_{y}$. The above model is valid only when all axles are on the ground. When one or more axle lifts off, the model is no longer valid and needs to be modified. When an axle lifts off, it does not contribute any restoring moment under increasing $\phi_{u}$. In other words, once $\phi_{u}$ is greater than the tire-lift-off angle, the restoring moment supplied by the tire saturates at $F_{z} \cdot T \cdot \phi_{u}$. The state space realization of the modified model is shown as follows:

$$
\dot{x}=A \cdot x+B \cdot u
$$

The augmented input vector $u=\left[\begin{array}{lll}a_{y} & \tilde{\phi}_{u 1} & \tilde{\phi}_{u 2}\end{array}\right]^{T}$ and $B=\left[\begin{array}{lll}b & c & d\end{array}\right]$, where

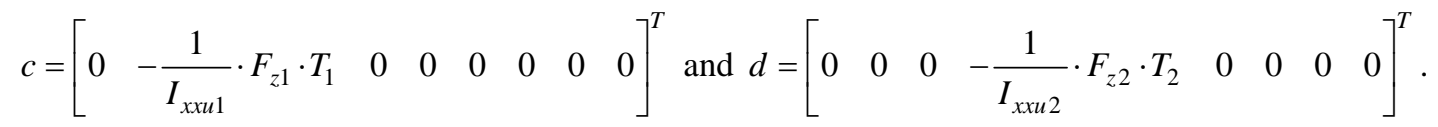

The state matrix $A$ also changes (switches) when axle lift-off occurs. The following two rules describe the modifications for matrix $A$ and the augmented input vector $u$.

(1) If $\phi_{u 1}>\phi_{u 1 \_l i f t-o f f}$, then $a_{21}=\frac{1}{I_{x x u 1}} \cdot\left[F_{u 1} \cdot\left(h_{r 1}-R_{t 1}\right)-K_{\phi s 1}+F_{z 1} \cdot R_{t 1}\right]$ and $\tilde{\phi}_{u 1}=\operatorname{sign}\left(\phi_{u 1}\right)$.

Else, then $a_{21}=\frac{1}{I_{x x u 1}} \cdot\left[F_{u 1} \cdot\left(h_{r 1}-R_{t 1}\right)-2 K_{t 1} \cdot T_{1}^{2}-K_{\phi s 1}+F_{z 1} \cdot R_{t 1}\right]$ and $\tilde{\phi}_{u 1}=0$. 
(2) If $\phi_{u 2}>\phi_{u 2 \_l i f t-o f f}$, then $a_{43}=\frac{1}{I_{x x u 2}} \cdot\left[F_{u 2} \cdot\left(h_{r 2}-R_{t 2}\right)-K_{\phi s 2}+F_{z 2} \cdot R_{t 2}\right]$ and $\tilde{\phi}_{u 2}=\operatorname{sign}\left(\phi_{u 2}\right)$.

Else, then $a_{43}=\frac{1}{I_{x x u 2}} \cdot\left[F_{u 2} \cdot\left(h_{r 2}-R_{t 2}\right)-2 K_{t 2} \cdot T_{2}^{2}-K_{\phi s 2}+F_{z 2} \cdot R_{t 2}\right]$ and $\tilde{\phi}_{u 2}=0$.

\section{Appendix B}

The parameters of the driver model are defined and their values are given in the following.

$U_{0} \quad$ Vehicle longitudinal speed (ft/sec)

$\tau_{\psi} \quad$ Time delay, $0.2 \mathrm{sec}$

$\tau_{r} \quad$ Time delay, $0.2 \mathrm{sec}$

$\tau_{a} \quad$ Time delay, $0.2 \mathrm{sec}$

$\varsigma_{n} \quad$ Damping ratio of neuromuscular dynamics, 0.5

$\omega_{n} \quad$ Natural frequency of neuromuscular dynamics, $20 \mathrm{rad} / \mathrm{sec}$

$K^{\prime} \quad$ Trim constant, $0.5 \mathrm{sec}^{-1}$

$T_{p} \quad$ Preview time, $1.25 \mathrm{sec}$

$K_{c} \quad$ The equivalent gain of the vehicle dynamics from steering input $\delta_{s w}$ to yaw rate $r$ at speed $U_{0}$

$T_{e q} \quad$ The equivalent first order lag of the vehicle dynamics from steering input $\delta_{s w}$ to yaw rate $\mathrm{r}$ at speed $U_{0}$

$\omega_{c} \quad$ Crossover frequency, $3 \mathrm{rad} / \mathrm{sec}$

$x_{a} \quad$ Look ahead distance, $x_{a}=U_{0} / A \mathrm{ft}$

$K_{y e} \quad$ Lane position trim gain, $K_{y e}=2 K^{\prime} / x_{z}^{2} \mathrm{ft}^{-2}-\mathrm{sec}^{-1}$

$K_{\psi} \quad$ Curvature feedback control gain, $K_{\psi}=\omega_{c} U_{0} / K_{c}$ rad-ft $/ \mathrm{sec}$

$K_{m} \quad$ Motion feedback gain, $K_{m}=T_{e q} K_{\psi} / U_{0}$ sec 